\title{
Analysis of nucleosome positioning determined by DNA helix curvature in the human genome
}

\author{
Hongde Liu, Xueye Duan, Shuangxin Yu, Xiao Sun ${ }^{*}$
}

\begin{abstract}
Background: Nucleosome positioning has an important role in gene regulation. However, dynamic positioning in vivo casts doubt on the reliability of predictions based on DNA sequence characteristics. What role does sequence-dependent positioning play? In this paper, using a curvature profile model, nucleosomes are predicted in the human genome and patterns of nucleosomes near some key sites are investigated.

Results: Curvature profiling revealed that in the vicinity of a transcription start site, there is also a nucleosome-free region. Near transcription factor binding sites, curvature profiling showed a trough, indicating nucleosome depletion. The trough of the curvature profile corresponds well to the high binding scores of transcription factors. Moreover, our analysis suggests that nucleosome positioning has a selective protection role. Target sites of miRNAs are occupied by nucleosomes, while single nucleotide polymorphism sites are depleted of nucleosomes.
\end{abstract}

Conclusions: The results indicate that DNA sequences play an important role in nucleosome positioning, and the positioning is important not only in gene regulation, but also in genetic variation and miRNA functions.

\section{Background}

Nucleosome positioning refers to the position of a DNA helix with respect to the histone core [1]. Positioning has important roles in gene regulation, because packing DNA into nucleosomes can limit the accessibility of the sequences [2-4]. High-resolution genome-wide nucleosome maps are now available for the genomes of yeast, worms, flies, and humans [2,5-7]. Studies of these nucleosome position datasets have revealed some interesting characteristics, especially for promoter sequences. A typical nucleosome-free region (NFR) is near the transcription start site (TSS) and is followed by a wellpositioned nucleosome [4]. Low nucleosome occupancy is a significant feature of a functional transcription factor binding site (TFBS) [8].

At the same time, computational predictions using DNA sequence information have also advanced. Since the report of the nucleosome positioning code (an $\sim 10$ bp repeating pattern of dinucleotides AA-TT-TA/GC) in yeast [9], some models for predicting nucleosomes have been developed using DNA sequence properties, such as dinucleotide periodicity, and structural information of

\footnotetext{
* Correspondence: xsun@seu.edu.cn

State Key Laboratory of Bioelectronics, Southeast University, Nanjing 210096, China
}

(C) 2011 Liu et al; licensee BioMed Central Ltd. This is an Open Access article distributed under the terms of the Creative Commons Attribution License (http://creativecommons.org/licenses/by/2.0), which permits unrestricted use, distribution, and reproduction in any medium, provided the original work is properly cited. the DNA helix $[5,7,10-13]$. The successful predictions suggest that DNA sequences partly encode nucleosomes themselves, although some deviations are observed between the predicted and the experimentally determined positions $[4,9]$. On further investigation, it was realized that dynamic positioning is a general rule in cells. Dynamic remodelling of one or two nucleosomes was revealed in yeast promoters [14]. Nucleosome reorganization of a gene might result from a cell-specific change or a condition-dependent change $[15,16]$. For cells from the same cell line, the first nucleosome downstream of the TSS exhibits differential positioning in active and silent genes, and such nucleosome reorganization can be induced in resting T-cells [2]. Relative positioning was also found to be a general characteristic in Caenorhabditis elegans [6].

Such variations of nucleosome positions in vivo cast doubt on the reliability of predictions based on DNA sequence characteristics [4,6]. Moreover, a recent work in yeast showed that there is no genome code in nucleosome positioning; even intrinsic histone-DNA interactions are not the major determinant [17]. Also, the mechanism by which DNA sequences guide nucleosomes positions is different between $S$. pombe and S. cerevisiae [18]. Nucleosome organization at the 3' 
end of genes conforms to the principles of statistical positioning [19].

However, some factors should be noted. Firstly, the 10-bp periodicity of dinucleotides AA-TT-TA/GC, which is identified as a positioning code in yeast, is also found in C. elegans, flies, and humans [9,20-24]. Secondly, strikingly similar features, including the NFR near the TSS, and the uniform spacing of internucleosomes downstream of the TSS, are observed both in the predicted data and in the experimentally determined data $[5,10,11]$. Low nucleosome occupancy is encoded around functional transcription factor binding sites $[8,9])$. Thirdly, some sequence-dependent models are suitable for predicting nucleosome positions in multiple genomes without additional information [7,11]. In addition, the chromatin remodelling complex can establish specific local chromatin structures by reading out DNA features and targeting nucleosomes to specific positions [25]. All of the above highlight the importance of sequence preferences in positioning.

In this paper, using the curvature profile, a new model based on the curvature pattern of nucleosomal DNA, nucleosomes positions were predicted for the human genome. Patterns of nucleosomes near interesting sites, including TSSs, TFBSs, single nucleotide polymorphism (SNP) sites, and target sites of miRNAs, were thoroughly investigated. The results also demonstrated the important roles of DNA sequences in determining nucleosomes. Moreover, we revealed that nucleosomes are not only functional in gene regulation, but also in genetic variation.

\section{Results and Discussion}

Predictions of nucleosome positions and the estimation of sequence-dependence

Nucleosomes positions were predicted by recognizing the curvature pattern of the core DNA helix (see methods). The reconstructed curvature pattern of a nucleosome is shown in Figure 1. To test how representative the pattern derived from the crystal structures was, 634 well-positioned (ratio of signal to noise $>100$ ) nucleosome DNA sequences

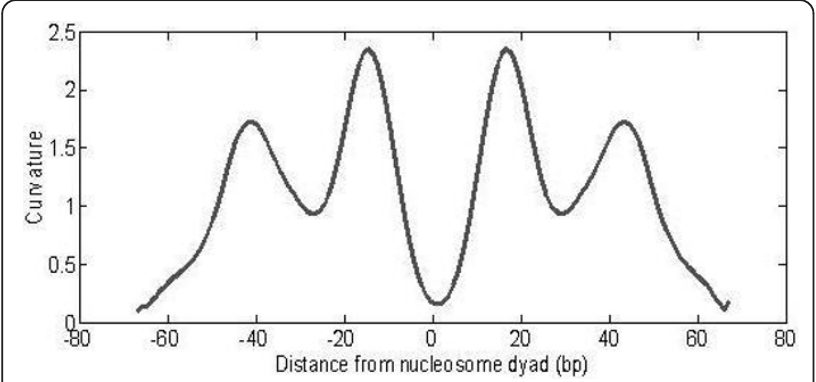

Figure 1 Curvature pattern of a nucleosomal DNA helix. were collected from Zhao et al.'s experimental dataset [2]. An averaged curvature curve of the 634 sequences resembles the pattern in shape (Additional file 1: Figure s1), indicating the pattern represents a canonical curvature of a nucleosomal DNA helix.

Using the curvature profile, nucleosomes positions were predicted for human chromosome 20. A waveletbased algorithm MSCWT [26] was employed in detecting exact dyad positions (Additional file 1: Figure s2). In the curvature profile and Kaplan et al.'s predictions, the averaged centre-to-centre distance of neighbour nucleosomes is $\sim 190 \mathrm{bp}$ (Additional file 1: Figure s3), close to value of $185 \mathrm{bp}$ in the literatures [2,4]. For Zhao et al.'s experimental dataset, due to the scarcity of the coverage, this value is $245 \mathrm{bp}$, which actually indicates nucleosomal repeats rather than the average distance.

A quantitative comparison between the predicted and the experimentally determined nucleosomes positions were carried out by measuring the distance between respective dyad positions (see Methods). More than 53\% of the experimentally determined nucleosomes were predicted by curvature profile, with a 40-bp deviation (Figure 2). Using the experimental data as a standard, the curvature profile shows a slightly higher performance than Kaplan et al.'s model, especially for a low deviation $(<25 \mathrm{bp})$. Importantly, both the curvature profile and Kaplan et al.'s model has a much higher matching ratio for the experimental data than random positioning does. In comparison with the model nuScore [13] on a 50k-bp sequence, the curvature profile exhibits a comparable performance (Additional file 1: Table s6). Additionally, nucleosomes have a good match in activated and resting $\mathrm{CD} 4^{+} \mathrm{T}$ cells $(65 \%$, deviation > $35 \mathrm{bp}$ ) (Figure 2), suggesting that most nucleosomes do not change in either type of cell; only a small number of nucleosomes, such as the first nucleosome downstream of a TSS [2], exhibit different position.

Figure 3 and Figure s5 (Additional file 1) demonstrate the predictions for two arbitrarily selected DNA sequences. The positive accuracy of the curvature profile is more than $55 \%$, with a deviation $<30$ bp (Additional file 1: Table s7). Moreover, the curvature profile exactly locates most of the TSSs and TFBSs in NFRs. This indicates that the curvature profile has a good capacity for predicting nucleosome positions, especially for key functional sites.

The curvature profile is based on the curvature characteristics of nucleosomal DNA, and is therefore sequence dependent. Subsequently, the curvature-dependent degree of nucleosome positioning was estimated using the nucleosome occupancy ratio of hexanucleotides in the whole of human chromosome 20. The correlation between occupancy ratio of the predictions and the experimental data was 0.6123 (Figure 4). It should be 


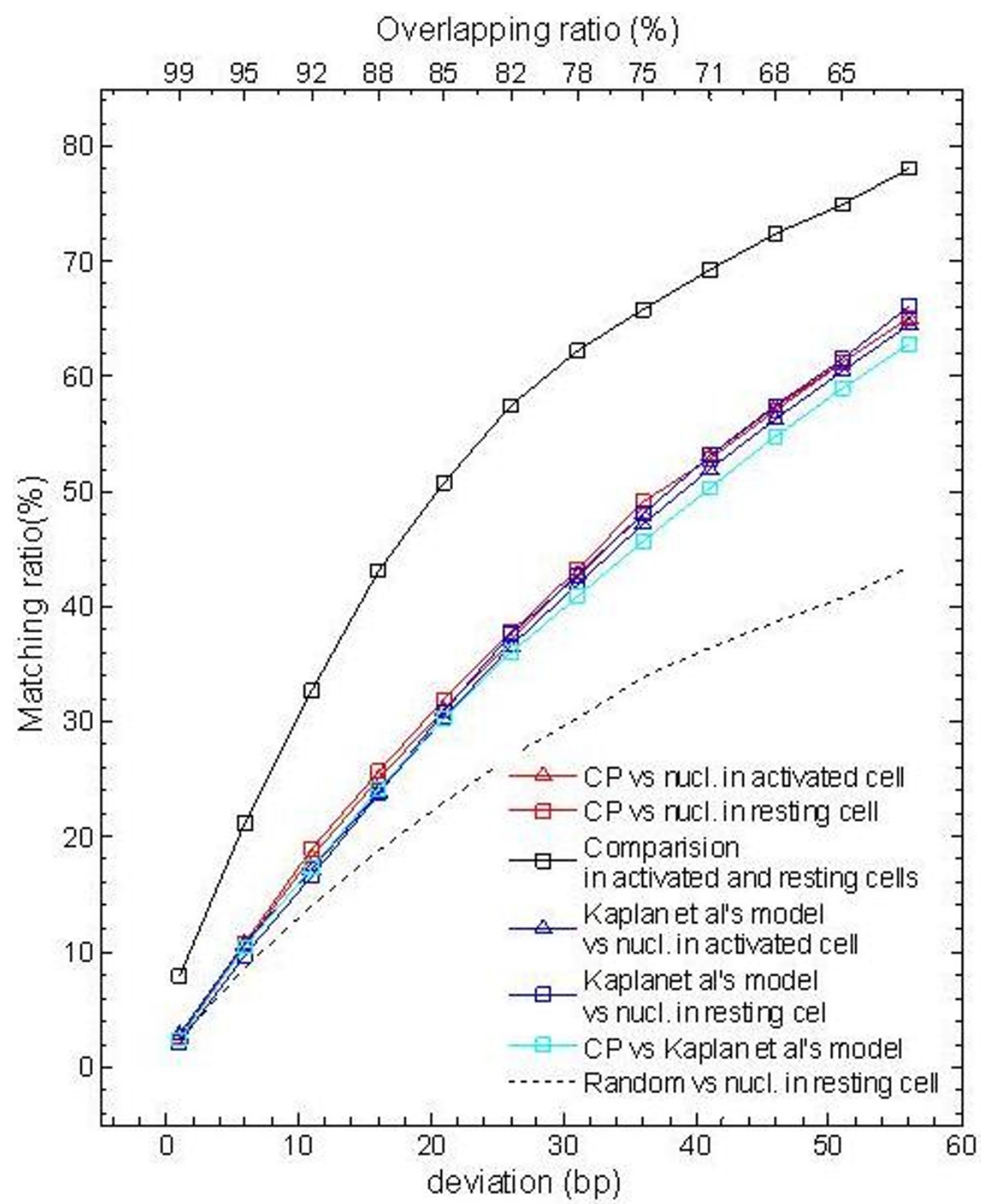

Figure 2 Performance of the curvature profile for nucleosome position prediction on human chromosome 20. Given a deviation, the matching ratio is a ratio of matched nucleosomes to all experimentally determined nucleosomes. Overlapping ratio is [2(73-deviation)+ deviation]/ 147 , indicating the degree of overlap between the predicted and the experimentally determined nucleosomes. "CP" indicates curvature profile.

pointed out that the curvature profile in fact reflects an indirect measurement of nucleosome position. Thus, the dependence in this study only reflects the influence of the curvature of the DNA helix on nucleosome positioning. We speculate that DNA sequences might encode a default arrangement of nucleosomes and that reorganization of nucleosomes in vivo is based on this default arrangement.
Nucleosome distribution in protein-coding promoters and independent miRNA promoters

Nucleosome positioning at promoters has been extensively investigated because of its role in occluding binding sites. A typical NFR locates around a TSS, which allows the binding sites to be exposed to the pre-initiation complex (PIC) $[2,5,9,11,16]$. The nucleosomes flanking the NFR also provide a steric match for the complex. 


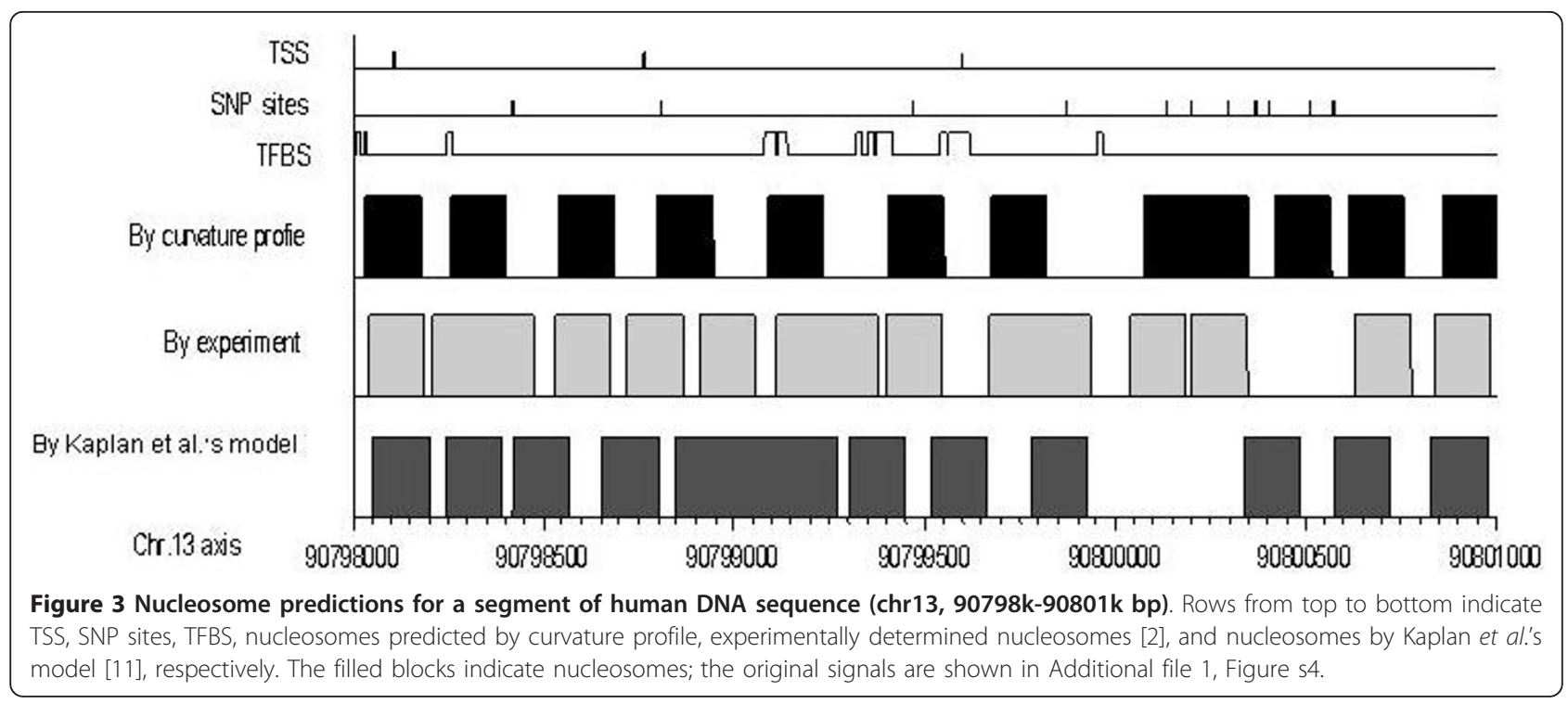

In this study, the averaged curvature profile (fine black line in Figure 5A) results in an $\sim 150$-bp NFR around the TSS of 3571 protein-coding genes, which is consistent with previous reports $[2,5,9,11,16]$. The NFR is also

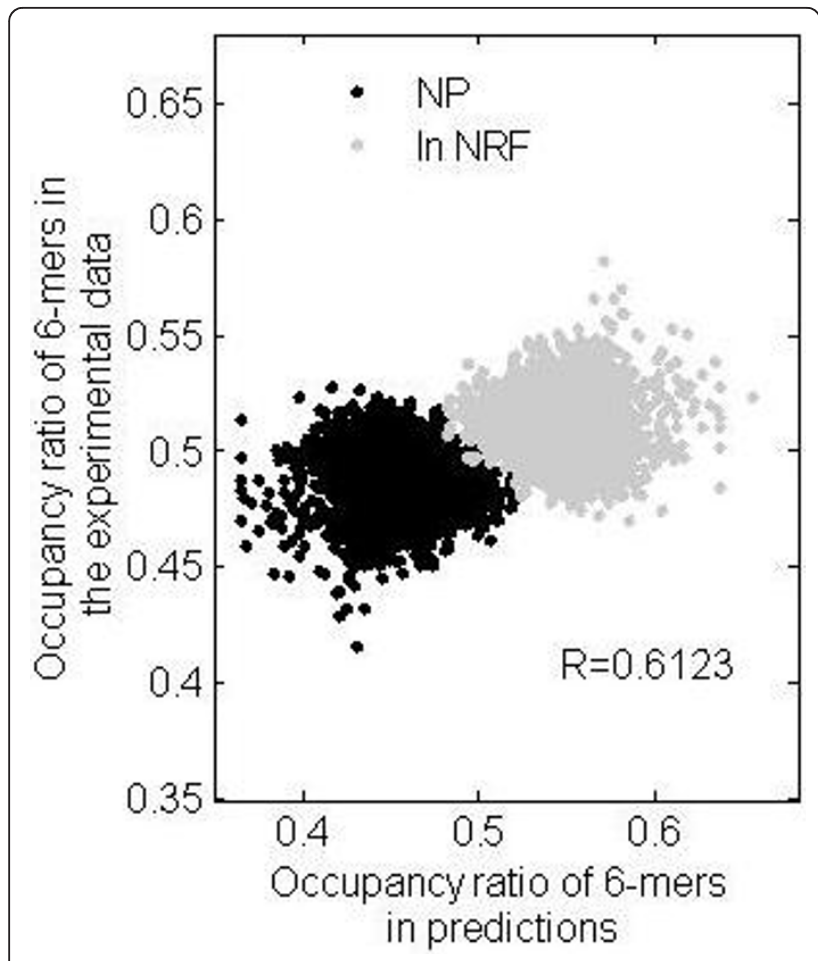

Figure 4 Estimation of curvature-dependent degree of nucleosome positioning. Black and grey dots represent the occupancy ratios and the nucleosome-free ratios of 4096 6-mer nucleotides, respectively. The estimated correlation coefficient is 0.6123; NP, nucleosome positioning; NFR, nucleosome-free region. revealed by Zhao et al.'s experimental data in activated $\mathrm{CD} 4^{+} \mathrm{T}$ cells (grey line in Figure 5A). However, not all TSSs are nucleosome free in vivo. Using Zhao et al.'s experimental data in the range of $-150 \mathrm{bp}$ to $50 \mathrm{bp}$ from the TSS, 3571 TSSs were divided into two classes by a k-means clustering method. This resulted in 1080 occupied TSSs (class I) and 2491 nucleosome-free TSSs (class II). Both the curvature profile and the experimental data are consistent with nucleosome depletion at class II TSSs (Figure 5C). Around the class I TSSs, no distinct positioning signal is observed in the curvature profile (Figure 5B), while a positioned nucleosome is suggested by the experiment data. This difference between the prediction and the experiment indicates that positioning is not completely determined by DNA sequences in vivo. The trough near the TSS in Figure $5 \mathrm{~B}$ is slightly higher than that in Figure $5 \mathrm{C}$, suggesting that a minority of class I TSSs are occupied by DNA sequence-encoded nucleosomes. As shown above, the nucleosome-free state is the default configuration, partly determined by DNA sequences at a TSS; however, in vivo, due to the function of the remodelling complexes, some of TSSs are occupied. This is why there are differences between the prediction and the experiment.

We computed the dinucleotide distribution near all the TSSs. Fraction of WW (W = A or T) dinucleotides decreased in a broad range near the TSS (Additional file 1: Figure s6A), Interestingly, in the range of $\sim 100 \mathrm{bp}$ upstream of the TSS, WW shows a sharp increase (Additional file 1: Figure s6A), also corresponding to an increase of poly (dA:dT) (Additional file 1: Figure s6B). We inferred that the increased poly (dA:dT) is associated with the NFR near the TSS, because the poly 


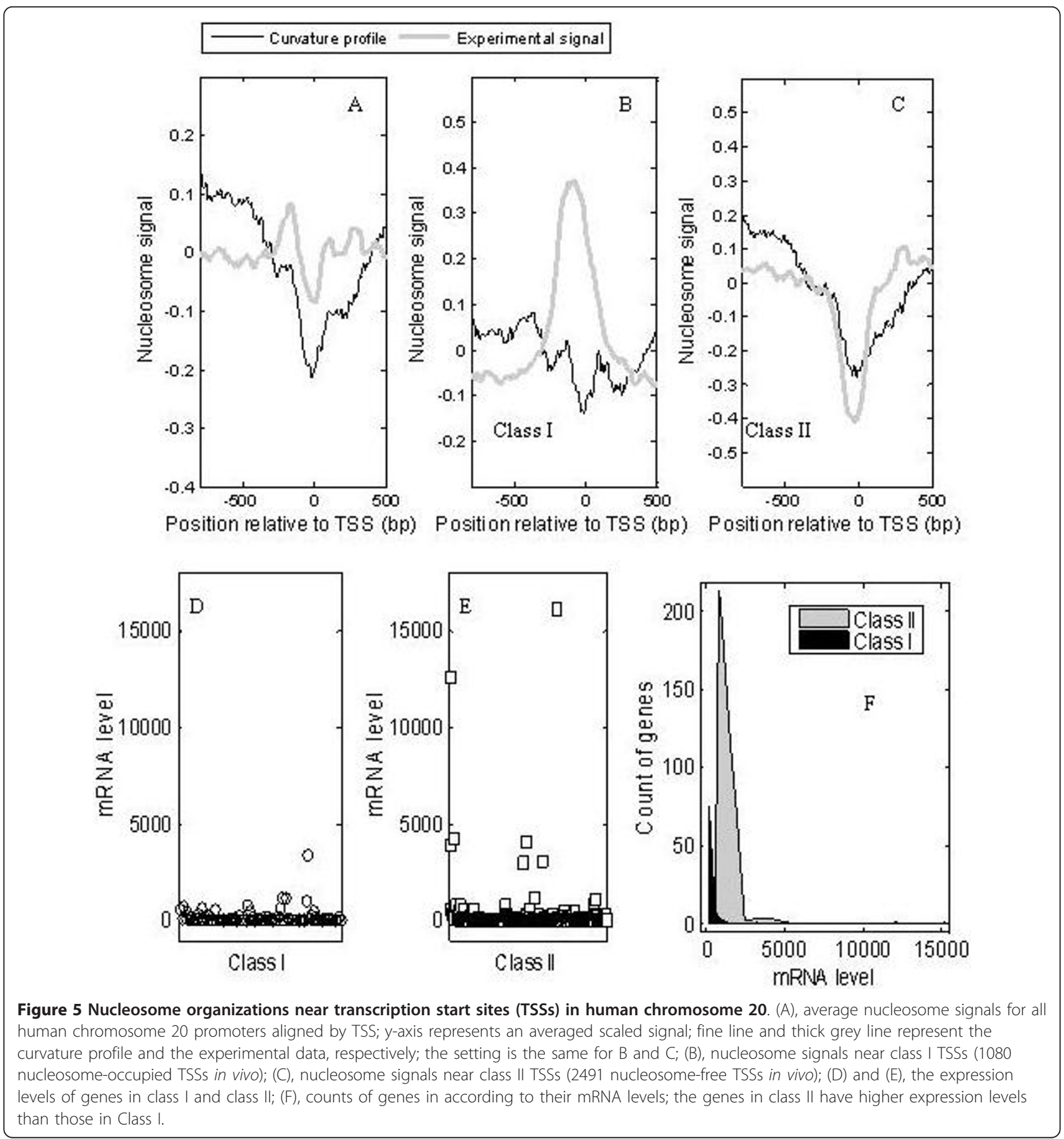

(dA:dT) disfavors nucleosome formation $[17,18]$. The results suggest that DNA sequence influences nucleosome positioning.

Taking these results together, DNA sequences partly encode a default NFR around a TSS. Due to the requirement of gene expression in vivo, some TSSs are occupied through chromatin remodelling, while others are still in NFRs. The positioned nucleosome at a TSS can block the binding sites of the pre-initiation complex, implying that a TSS-occupied gene should exhibit a lower expression level. This implication was verified by examining mRNA levels using gene expression data in $\mathrm{CD} 4^{+} \mathrm{T}$ cells [27]. As shown in Figure 5F, the mRNA levels of TSS-occupied genes (class I) are lower than those of nucleosome-free TSS genes (class II). A similar result is observed using Zhao et al.'s gene expression 
data in activated $\mathrm{CD}^{+} \mathrm{T}$ cells (see Additional file 1: Figure s7). The results indicate that curvature-dependent nucleosomes partly determine gene expression level.

Subsequently, nucleosome positioning at miRNA promoters was investigated. We predicted nucleosomes of miRNA promoters with the curvature profile and Kaplan et al.'s model [11]. Surprisingly, both models give a unanimous result (Figure 6). The mean of the deviations is less than $25 \mathrm{bp}$. Moreover, the TSSs of miRNA promoters were exactly located in NFRs, which is similar to protein-coding promoters. Two wellpositioned nucleosomes flank the NFR. The results suggest that nucleosome positioning is involved in the regulation of protein-coding genes and of miRNA genes.

It has been reported that there is a low nucleosome level near a TFBS [8]. This feature of nucleosome organization was also observed in the curvature profile. In Figure 7A, a trough of nucleosome level is observed near TFBSs, suggesting open chromatin is indispensable to the binding of transcription factors. Due to the low resolution of the curvature profile, the trough in the curvature profile is broader than that determined by the experimental data.

As TFBSs are most frequently located in the NFRs of promoters, the NFR will correspond to the region with a high density of TFBSs (high scanning score of TFBS) in promoters $[8,11]$. This hypothesis was verified for both protein-coding promoters and independent miRNA promoters, by examining the relationship between the curvature profile and the average binding score profile of 64 human transcription factors (TFs) in the promoters. Eight protein-coding promoters and thirteen human independent miRNA promoters were used (see methods). As expected, the trough of the curvature profile corresponds well to the high binding scores of TFs, and regions with high levels of positioning signal (indicating nucleosome occupancy) have correspondingly low binding scores (see Figure 7). The mutually antagonistic relationship is obvious in the region of $-600 \mathrm{bp}$ to $400 \mathrm{bp}$ for protein-coding promoters (Figure $7 \mathrm{~B}$ ), and $-500 \mathrm{bp}$ to $100 \mathrm{bp}$ for independent miRNA promoters (Figure 7C). Outside of these regions, the relationship broke down, indicating that the regions mentioned above are important for TF binding. Despite the small number of genes used, the results indicate that a positioned nucleosome limits TF binding for both protein-coding promoters and miRNA promoters. Most importantly, it strongly suggests that the DNA sequences affect transcription, not only by providing special binding sites, but also by influencing nucleosome positioning.

Recent findings suggest that the mechanisms by which DNA sequences affect nucleosome positioning is distinct in some species $[6,18,19,27]$; and the choice of the periodical dinucleotides differs considerably from one organism to another [28], indicating the difficulty in finding a universal positioning code. However, it was
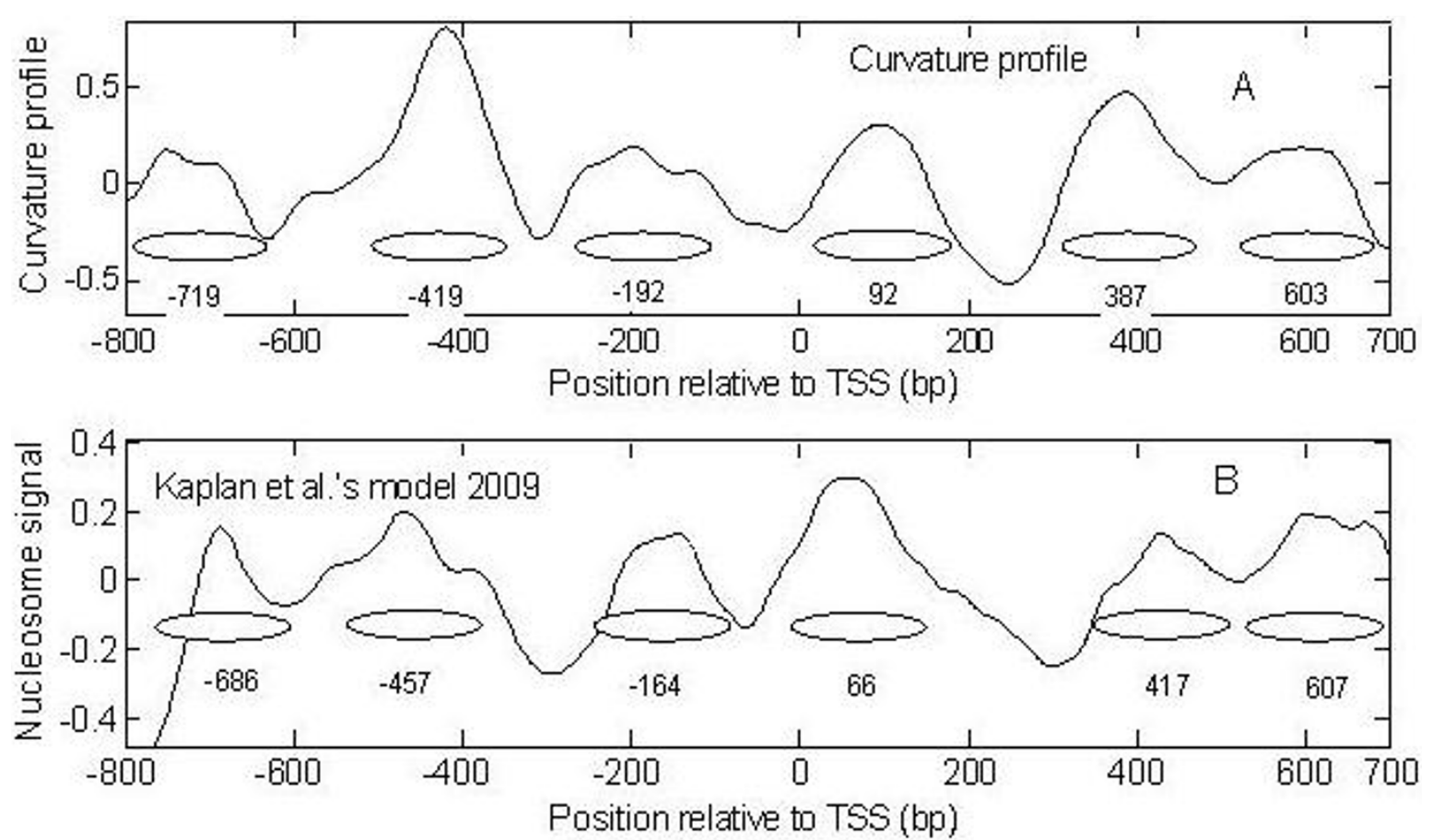

Figure 6 Average nucleosome organization in the vicinity of an miRNA TSS. (A), curvature profile; (B), Kaplan et al.'s model prediction [11]; ellipses indicate nucleosomes; the numbers below them indicate the dyad position relative to transcription start sites. 

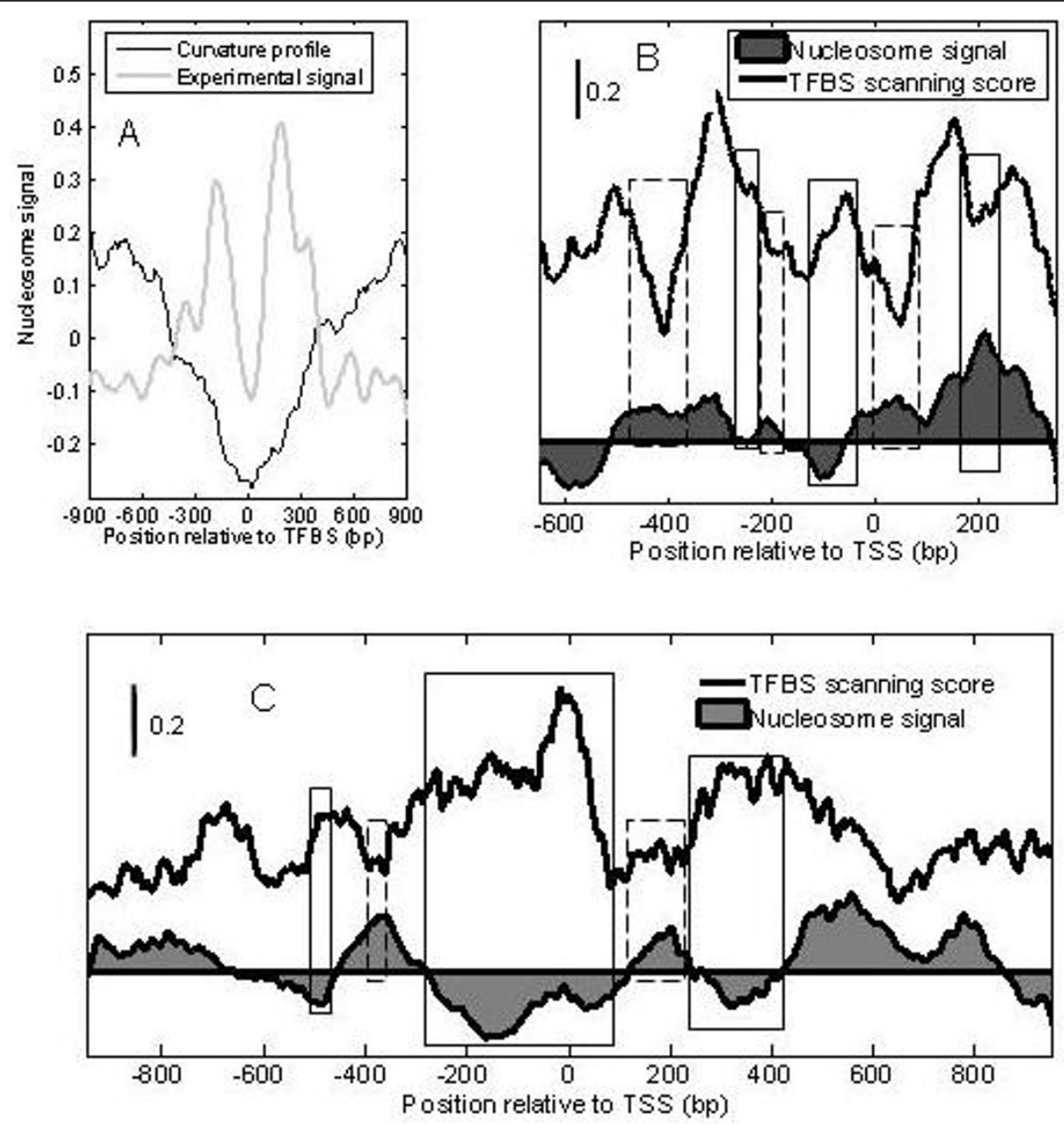

Figure 7 Patterns of nucleosomes near transcription factor binding sites (TFBSs). (A), patterns of nucleosomes near transcription factor binding sites (TFBS); (B) and (C), the relationship between nucleosome positioning and the distribution of TFBS, (B) for 13 independent MiRNA promoters [37], (C) for eight protein-coding genes' promoters (ZBED4, ZNF378, PIK4CA, EWSR1, SMC1L2, NF2, ARFGAP3, KIAA0542) [38]; the bold black lines represent the distribution of TFBS (the average binding score profile of 64 TFs, see methods), the area with shading are averaged curvature profile, in which reference lines are cut-off lines. The vertical blocks highlight the correspondences between NFR and the distribution of TFBS.

observed that some important characteristics (such as NFRs) revealed by DNA sequence-based models are coincident with those observed in vivo [5,7-13]. DNA structure-related periodicity $(\sim 10-\mathrm{bp})$ is suggested in yeast, fly, worm, and human genomes [9,20-24]. These indicate that DNA sequence is one of the contributors to nucleosome positioning. The detail of how DNA sequence affects nucleosome positioning requires further investigation. One should be very carefully in using features derived from one organism to predict nucleosomes in other organisms. Recent studies showed that in RSCdepleted cells, nucleosomes move toward predicted sites [29]. Taken together, we speculate that DNA sequences partly determine a default pattern of nucleosomes positions, on which nucleosome reorganization is based. Thus, feature-based models can provide a view of nucleosome configuration determined by DNA sequences, and assist in finding certain key sites (such as TSSs) when an experimental dataset is absent.

\section{Genetic variation and nucleosome positioning}

Patterns of nucleosomes near SNP sites were investigated (see Figure 8). SNP sites are grouped into four classes, single, insertion/deletion (indels), insertion, and deletion. Near the sites of indels, insertions, and deletions, the curvature profile shows a large trough (Figure 8B-D), indicating such mutation events favor linker-DNA. Importantly, a similar result was also observed using the experimental data. Although the trough near the single SNP sites is not as low as that near the other types of SNP (Figure 8A), 

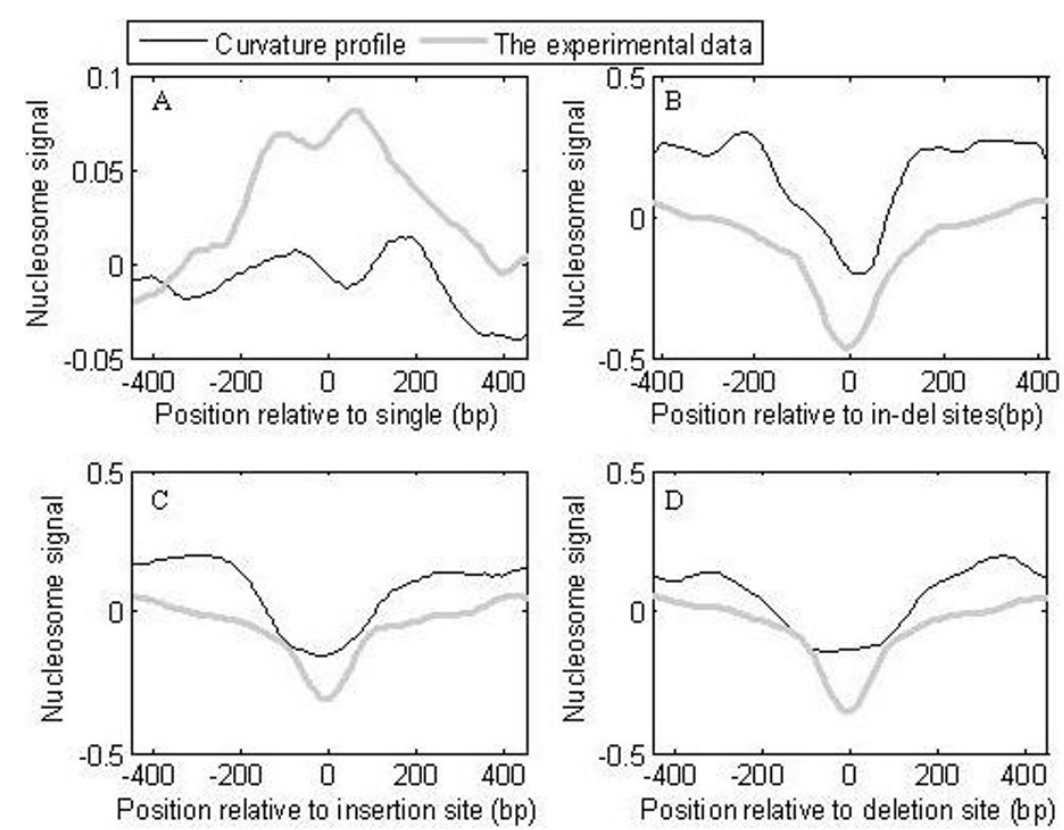

Figure 8 Patterns of nucleosomes near SNP sites. (A), single nucleotide variation (single); (B), insertions/deletions (in-del); (C), insertion SNP; (D), deletion SNP.

nucleosome depletion is obvious. In the genome of Oryzias latipes, genetic variation downstream of a TSS occurs with an 200-bp periodicity, and the insertion/deletion of more than $1 \mathrm{bp}$ frequently occurs in the linker-DNA region [30], which is consistent with our findings in humans. Moreover, we found that nucleosomes are also depleted near SNP sites in the dog genome (Additional file 1: Figure s8).

The above results suggest a link between nucleosome positioning and genetic variation. Nucleosome positioning has a protective role for splice sites [31]. Our findings show the opposite effect on sites depleted of nucleosomes. The sites without the nucleosomes are prone to mutations. In fact, a well-positioned nucleosome is observed at the target sites of miRNAs, protecting the key sites (see below).

\section{Nucleosome positioning at target sites of miRNA}

Figure 9A shows the positioning pattern of nucleosomes in the vicinity of miRNA target sites. A well-positioned nucleosome is observed at the target sites in the curvature profile. The curve of the experimental data is very similar to the curvature profile in the region far from the target sites. However, near the centre of the target sites, the experimental data gives a great valley while the curvature profile shows a peak. The similarity of two curves in the region either side of the target sites suggests that we cannot simply attribute the opposing patterns (the trough in the experimental data and the peak in curvature profile near the centre of target site) to the inaccuracy of the curvature profile. We suspect that some unknown factors prevent Zhao et al.'s experiment from successfully detecting the nucleosomes positioned at the target sites. Nucleosomes have a protective role for some special sites, such as splice sites [31]. miRNAs are known to be involved in post-transcriptional regulation, by binding to the 3'-UTR region of an mRNA sequence using antisense base pairing and cleaving the target mRNA or repressing its translation into protein [32]. Mutations at miRNA target sites will result in recognition errors for miRNAs. Thus, it is essential to protect the target sites of miRNAs in the nucleolus. The curvature profile's results suggest that miRNA target sites are protected by nucleosomes.

Assuming that the target sites of miRNAs are protected, the accumulation of genetic variation in target sites should be at a low level. Thus, we examined the distribution of SNPs in miRNA target sites (see Figure 9B). In range of $-15 \mathrm{bp}$ to $10 \mathrm{bp}$ from the target sites, the SNP counts obviously decreases, indicating that the target sites are conserved in evolution. Taking into consideration the nucleosome depletion near SNP sites, it is thought that nucleosomes have a role in protecting target sites of miRNA.

As indicated above, nucleosome positioning protects some key sites, while allowing other sites to remain open. This selective protection facilitates both genome conservation and evolution. The patterns were revealed by curvature-dependent computations (the curvature profile). Therefore, genome sequences partly encode nucleosomes, and the latter allow mutations to occur at 


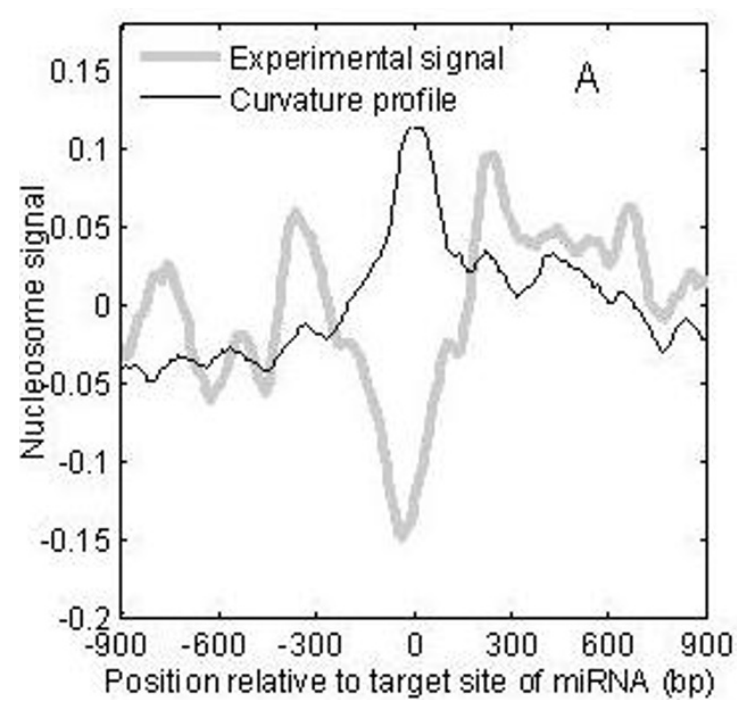

Figure 9 Nucleosome positioning at miRNA target sites. (A), patterns of nucleosomes near target sites of miRNAs; (B), distribution of SNPs in the vicinity of target sites of miRNAs in human chromosome 20.

some sites and protect other special sites. Thus, nucleosome positioning has roles in genetic variation.

\section{Conclusions}

In this study, human nucleosome positions were predicted with a curvature-dependent model; the curvature profile. The results indicate that the importance of DNA sequences in determining nucleosome positions, providing a default pattern. DNA sequence partly encodes an NFR near a TSS. In vivo, the TSSs of some genes are occupied by nucleosomes, and changes to nucleosome positioning will affect the genes' expression levels. In promoters, nucleosomes are depleted near TFBSs, and the distribution of TFBSs corresponds well with the NFRs. Moreover, a selective protection role of nucleosomes was revealed. SNP sites are enriched in NFRs, and miRNA target sites are associated with wellpositioned nucleosomes. Our results indicate the vital role of the DNA sequence in encoding nucleosomes, and that the functions of nucleosome positioning are probably involved in further biological processes.

\section{Methods}

\section{The prediction model}

In our previous work [23], we found that WW (W = A or T) dinucleotides of core DNA sequences showed smaller spacing ( 10.3 bp) at the two ends ( $50 \mathrm{bp})$ of a nucleosome, with larger ( 11.1 bp) spacing in the middle section ( $\sim 7 \mathrm{bp})$. In fact, this is consistent with the cutting periodicities of core DNA [1]. Correspondingly, core DNA helices showed greater bending at the two ends, with smaller curvatures in the middle section. Using these findings, we constructed two nucleosome prediction models,

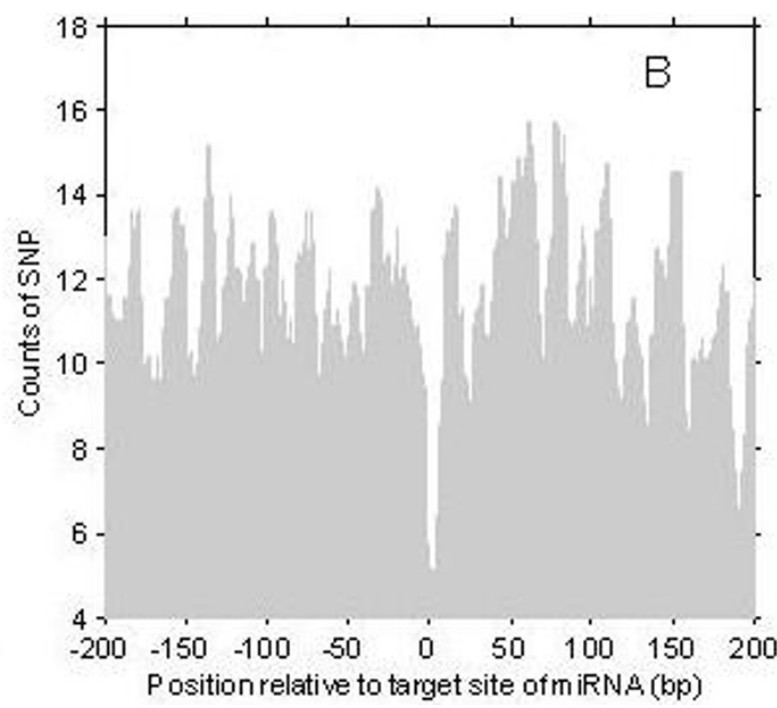

the periodicity profile and the curvature profile [23]. The periodicity profile has good resolution; however, due to wavelet transformation, it is time-consuming. The curvature profile is highly efficient in computation, but overlapping peaks and significant noise decrease its resolution and hinder the recognition of nucleosomes.

To improve the resolution of the curvature profile, the curvature pattern was reconstructed in this paper. Eighteen human nucleosomal DNA sequences (146 bp) were extracted from the crystal structure dataset of the nucleosomal DNA and histone proteins (Additional file 1: Table s1). The DNA curvatures of the nucleosomal sequences were estimated using the curvature vector $C$ (eq.1), which is calculated with a matrix of roll $\rho$ and tilt $\tau$ angles (Additional file 1: Table s2), obtained for the sixteen dinucleotide steps (eq.1) [33].

$$
C=v^{0}\left(n_{2}-n_{1}\right)^{-1} \sum_{j=n_{1}}^{n_{2}}\left(\rho_{j}-i \tau_{j}\right) \exp \left(\frac{2 \pi i j}{v^{0}}\right)
$$

where $v^{0}$ is the double-helix average periodicity (10.4 bp). The numbers $\left(\mathrm{n}_{2}-\mathrm{n}_{1}\right)$ represent the integration steps. The modulus of the vector represents the deviation from B-DNA. The results generated a uniform pattern of curvature for nucleosomal sequences (see Figure 1). The pattern is called the curvature pattern of nucleosomal DNA.

Given a DNA sequence, the curvature value is calculated at each position using eq.1. The whole curvature of the sequence is called the curvature curve. The nucleosome positions can be predicted from the convolution of the curvature curve and the curvature pattern 
signal (Figure 1). If a segment of the curve resembles the pattern signal, the convolution will peak at the corresponding position, indicating a nucleosome. The convoluted curvature curve is called the curvature profile (Additional file 1: section 1).

\section{Analysis of nucleosome positioning in the human genome}

Human genomic DNA sequences were retrieved from NCBI (http://www.ncbi.nlm.nih.gov/) (build 36.1), and nucleosomes were mapped using the curvature profile. To evaluate the predictions, the experimentally determined nucleosomes dataset of activated and resting $\mathrm{CD} 4^{+}$ T-cells was downloaded from Zhao's website (http://dir. nhlbi.nih.gov/papers/lmi/epigenomes/hgtcellnucleosomes. aspx) [2]. The dataset was used as the "standard" dataset for comparison and is called "the experimental data" in this paper. Zhao et al.'s dataset contains two columns for each chromosome, the first column indicates the genomic positions and the second shows scores of nucleosomes. A method based on wavelet transformation was used to detect the exact nucleosome positions from the series of scores (see below). Kaplan et al.'s predicted nucleosome dataset was downloaded from website (http://genie.weizmann.ac.il/software/nucleo_genomes.html) [11]. Comparisons between curvature profiles and Kaplan et al.'s model were carried out on human chromosome 20.

In the experimentally determined datasets, Kaplan et al.'s predictions, and the curvature profile, the dataset are a series of numbers. Thus, it is important to identify peaks positions to perform a quantitative comparison. Here, the maximal spectrum of continuous wavelet transformation (MSCWT) [26] (Additional file 1: section 2) was used to detect the dyad positions of nucleosomes from the raw signals of the curvature profiles and the experimentally determined nucleosomes dataset. A nucleosomal DNA was defined in a $147-$ bp DNA by extending $73 \mathrm{bp}$ in both directions (5' and 3') from each of the dyad positions. The dataset of the dyad positions is available on our website.

The dyad positions determined by the curvature profile were compared with that in Zhao et al.'s experimental data by measuring the distance between respective dyad positions. Given a deviation, the amount of matched nucleosomes was counted, and a matching ratio was estimated by dividing the amount of matched nucleosomes by the total number of experimentally determined nucleosomes. The deviation varied from 1 bp to $60 \mathrm{bp}$. The comparison was performed for human chromosome 20. To explore the dynamic nucleosome positioning, the experimental datasets from both activated and resting $\mathrm{CD} 4^{+} \mathrm{T}$ cells were used. Additionally, the performance of Kaplan et al.'s predictions was presented.

\section{Estimation of the sequence-dependence of nucleosome positioning}

The occupancy ratio for each 6-mer nucleotide was computed by dividing the counts of nucleosome-occupancy by the counts of the hexanucleotides in human chromosome 20. The procedure was performed for both the predicted nucleosomes and the experimentally determined nucleosomes. The sequence-dependence degree was then estimated by correlating the two sets of ratios.

\section{Pattern of nucleosomes near special sites}

More attention was paid to nucleosomes around special sites within the genome. Upstream and downstream sequences of TSSs, TFBSs [34], SNPs [35] and target sites of miRNAs [36] were extracted from UCSC (http://genome.ucsc.edu/). Information on miRNA promoters was extracted from the literature [37]. Details of these sequences are listed in Tables s3 and s4 (Additional file 1). The sequences were aligned by their special sites, and the nucleosome patterns were represented by averaged curvature profiles. To examine the effect of nucleosomes on gene expression in vivo, protein-coding TSSs were separated into two classes by a $k$-means clustering method using Zhao et al.'s experimental data [2] in a range of $150 \mathrm{bp}$ upstream to $50 \mathrm{bp}$ downstream of the TSS in activated $\mathrm{CD}^{+} \mathrm{T}$ cell. One class (class I) contained TSSs that are occupied by nucleosomes; the other (class II) contained nucleosome-free TSSs. A dataset of mRNA levels [27] was used to check the effect of nucleosomes on gene expression (Additional file 1: section 3).

To test whether nucleosomes limit the binding of transcription factors, the distribution of TFBSs was computed for both protein-coding promoters (ZBED4, ZNF378, PIK4CA, EWSR1, SMC1L2, NF2, ARFGAP3, and KIAA0542) [38] and independent miRNA promoters (bold and italic in Additional file 1, Table s4) [37]. We scanned the binding scores of 64 human TFs (Additional file 1: Table s5) on the promoter sequences. Each TF had a unique position weight matrix (PWM); scanning with PWM on a promoter sequence resulted in a binding score profile, which indicated the potential binding regions of the TF on the sequence. The distribution of TFBSs was represented by the average binding score profile of 64 TFs on all promoters. PWMs were obtained using JASPAR [39].

An online-prediction tool for the curvature profile is provided (http://www.gri.seu.edu.cn/icons).

\section{Additional material}

\footnotetext{
Additional file 1: Section 1 - The prediction model. Section 2

Detection of peak positions in curvature profiles, Zhao et al.'s dataset and Kaplan et al.'s predictions. Section 3 - Examining the expression level of TSS-occupied genes and TSS nucleosome-free genes. Table s1 - Eighteen
} 
crystal structure datasets of the DNA-histone proteins used to reconstruct the curvature characteristic. Table $s 2$ - Values of roll $\rho$ and tilt $\tau$ angles of sixteen dinucleotide steps. Table s3 - Details on sequences around transcription start sites (TSSs), single-nucleotide polymorphism (SNP) sites, target sites of miRNAs, start and stop codons, and boundaries of histone modifications. Table s4 - Three types of miRNAs in humans. Table s5 - Sixtyfour human transcription factors used in scanning. Table s6 - Comparison of performances of the curvature profile and nu-Score. Table s7 - Prediction performance of the curvature profiles in Figure 3 and s5. Table $s 8$ - Top 20 6-mer nucleotides that are favorable for nucleosomes and nucleosome-free regions. Figure s1 - Curvature pattern derived from 634 well-positioned nucleosome DNA sequences in the experimental dataset. Figure $s 2$ Identification of nucleosome dyad positions in DNA sequences from $8 \mathrm{kbp}$ to $28 \mathrm{k}$ bp of human chromosome 20. Figure s3 - Distribution of centre-tocentre distance of nucleosomes. Figure $\mathrm{s} 4$ - Predictions of nucleosomes for the segment from $90798 \mathrm{k}$ bp to $90801 \mathrm{k}$ bp of human chromosome 13. Figure 55 - Predictions of nucleosomes for a segment of human chromosome 17 (52269 k-52289 k bp). Figure s6 - (A) Faction distributions of WW (W = A ot T) dinucleotides and SS ( $=\mathrm{G}$ or $\mathrm{C})$ dinucleotides near 3571 transcription start sites; (B), fraction of poly (dA) and poly (dT); (C), fraction of poly (dG) and poly (dC). Figure s7-Gene expression levels (mRNA levels) for the occupied-TSS genes (class I) and the open-TSS (class II) in activated $\mathrm{CD}^{+} \mathrm{T}$ cells, the gene expression data is from Zhao et al's experiment (GEO accession number, GSE10437). Figure s8- Patterns of nucleosomes near SNP sites in the dog genome.

\section{Acknowledgements}

We thank the anonymous reviewers for their suggestions to improve the article's quality. This work was supported by the Natural Science Foundation of China (30800209,61073141), the Jiangsu Natural Science Foundation (SBK201022749), and the Leading Science Project of Southeast University of China.

\section{Authors' contributions}

HDL: model construction, data analysis, and paper preparation; SXY: web server construction; XYD: language revision and data analysis; XS: analysis of results. All authors have read and approved the final manuscript.

Received: 8 June 2010 Accepted: 27 January 2011

Published: 27 January 2011

\section{References}

1. Lewin B: Gene VIII. Prentice Hall 2004, Chap. 20.

2. Schones DE, Cui K, Cuddapah S, Roh TY, Barski A, Wang ZB, Wei G, Zhao KJ: Dynamic Regulation of Nucleosome Positioning in the Human Genome. Cell 2008, 132:887-898.

3. Henikoff S: Nucleosome destabilization in the epigenetic regulation of gene expression. Nat Rev Genet 2008, 9(1):15-26.

4. Jiang C, Pugh BF: Nucleosome positioning and gene regulation: advances through genomics. Nat Rev Genet 2009, 10(3):161-72.

5. Yuan GC, Liu JS: Genomic sequence is highly predictive of local nucleosome depletion. PLoS Comput Biol 2008, 4:e13.

6. Valouev A, Ichikawa J, Tonthat T, Stuart J, Ranade S, Peckham H, Zeng K, Malek JA, Costa G, McKernan K, Sidow A, Fire A, Johnson SM: A highresolution, nucleosome position map of $C$ elegans reveals a lack of universal sequence-dictated positioning. Genome Res 2008, 18(7):1051-1063

7. Miele V, Vaillant C, d'Aubenton-Carafa Y, Thermes C, Grange T: DNA physical properties determine nucleosome occupancy from yeast to fly. Nucl Acids Res 2008, 36:3746-3756.

8. Daenen F, Roy F, De Bleser J: Low nucleosome occupancy is encoded around functional human transcription factor binding sites. BMC Genomics 2008, 9:332-330.

9. Segal E, Mittendorf YF, Chen L, Thåström A, Field Y, Moore IK, Wang JZ, Widom J: A genomic code for nucleosome positioning. Nature 2006, 442(17):772-778.

10. loshikhes I, Albert I, Zanton SJ, Pugh BF: Nucleosome positions predicted through comparative genomics. Nat Genet 2006, 38(10):1210-1215.
11. Kaplan N, Moore IK, Mittendorf YF, Gossett AJ, Tillo D, Field Y, LeProust EM, Hughes TR, Lieb JD, Widom J, Segal E: The DNA-encoded nucleosome organization of a eukaryotic genome. Nature 2009, 458:362-366.

12. Morozov AV, Fortney K, Gaykalova AD, Studitsky MV, Widom J, Siggia ED: Using DNA mechanics to predict in vitro nucleosome positions and formation energies. Nucl Acids Res 2009, 37(14):4707-4722.

13. Tolstorukov MY, Choudhary V, Olson WK, Zhurkin VB, Park PJ: nuScore: a web-interface for nucleosome positioning predictions. Bioinformatics 2008, 24:1456-1458.

14. Shivaswamy S, Bhinge A, Zhao Y, Jones S, Hirst M, lyer VR: Dynamic remodeling of individual nucleosomes across a eukaryotic genome in response to transcriptional perturbation. PLOS Biol 2008, 6(3):e65.

15. Steven JP, Lis JT: Rapid, Transcription-Independent Loss of Nucleosomes over a Large Chromatin Domain at Hsp70 Loci. Cell 2008, 134(1):74-84.

16. Ozsolak F, Song JS, Liu XS, Fisher DE: High-throughput mapping of the chromatin structure of human promoters. Nat Biotech 2007, 25(2):244-248.

17. Zhang Y, Moqtaderi Z, Rattner PB, Euskirchen G, Snyder M, Kadonaga TJ, Liu XS, Struhl K: Intrinsic histone-DNA interactions are not the major determinant of nucleosome positions in vivo. Nat struct mol biol 2009, 16(8):847-853.

18. Lantermann AB, Straub T, Strålfors A, Yuan GC, Ekwall K, Korber P: Schizosaccharomyces pombe genome-wide nucleosome mapping reveals positioning mechanisms distinct from those of Saccharomyces cerevisiae. Nat Struct Mol Biol 2010, 17:251-257.

19. Mavrich TN, loshikhes IP, Venters BJ, Jiang CZ, Tomsho LP, Qi J, Schuster SC, Albert I, Pugh BF: A barrier nucleosome model for statistical positioning of nucleosomes throughout the yeast genome. Genome Res 2008, 18(7):1073-83.

20. Herael $\mathrm{H}$, Weiss $\mathrm{O}$, Trifonov EN: 10-11 bp periodicities in complete genomes reflect protein structure and DNA folding. Bioinformatics 1999, 15(3):187-193.

21. Schieg P, Herzel H: Periodicities of 10-11 bp as indicators of the supercoiled state of genomic DNA. J Mol Biol 2004, 234:891-901.

22. Fukushima A, Ikemura T, Kinouchi M, Oshima T, Kudo Y, Mori H, Kanaya S: Periodicity in prokaryotic and eukaryotic genomes identified by power spectrum analysis. Gene 2002, 300:203-211.

23. Liu HD, Wu JS, Xie JM, Yang XN, Lu ZH, Sun X: Characteristics of nucleosome core DNA and their applications in predicting nucleosome positions. Biophys J 2008, 94(12):4597-4604.

24. Salih F, Salih B, Trifonov EN: Sequence structure of hidden 104-base repeat in the nucleosomes of C elegans. J Biomol Struct Dyn 2008, 26:273-282.

25. Rippe K, Schrader A, Riede P, Strohner R, Lehmann E, Längst G: DNA sequence- and conformation-directed positioning of nucleosomes by chromatin-remodeling complexes. Proc Natl Acad Sci USA 2007, 104(40):15635-15640

26. Lu XQ, Liu HD, Xue ZH, Wang XQ: Maximum Spectrum of Continuous Wavelet Transform and Its Application in Resolving an Overlapped Signal. J Chem Inf Comput Sci 2004, 44:1228-1237.

27. Andrew IS, Wiltshire T, Batalov S, Lapp H, Ching KA, Block D, Zhang J, Soden R, Hayakawa M, Kreiman G, Cooke MP, Walker JR, Hogenesch JB: A gene atlas of the mouse and human protein-encoding transcriptomes. Proc Natl Acad Sci USA 2004, 101(16):6062-6067.

28. Bettecken T, Trifonov EN: Repertoires of the Nucleosome-Positioning Dinucleotides. PLOS ONE 2009, 4(11):e7654.

29. Hartley PD, Madhani HD: Mechanisms that Specify Promoter Nucleosome Location and Identity. Cell 2009, 137:445-458.

30. Sasaki S, Mello CC, Shimada A, Nakatani Y, Hashimoto S, Ogawa M, Matsushima K, Gu SGP, Kasahara M, Ahsan B, Sasaki A, Saito T, Suzuki Y, Sugano S, Kohara Y, Takeda H, Fire A, Morishita S: Chromatin-Associated Periodicity in Genetic Variation Downstream of Transcriptional Start Sites. Science 2009, 323(5912):401-404.

31. Kogan S, Trifonov EN: Gene splice sites correlate with nucleosome positions. Gene 2005, 6(352):57-62.

32. Pasquinelli AE, Hunter $\mathrm{S}$, Bracht J: MicroRNAs: A developing story. Curr Opin Genet Dev 2005, 15:200-205.

33. Cacchione S, Santis PD, Foti D, Palleschi A, Savino M: Periodical polydeoxynucleotides and DNA curvature. Biochemistry 1989, 28:8706-8713.

34. Johnson DS, Mortazavi A, Myers RM, Wold B: Genome-wide mapping of in vivo protein-DNA interactions. Science 2007, 316(5830):1497-502. 
35. Sherry ST, Ward MH, Kholodov M, Baker J, Phan L, Smigielski EM, Sirotkin K: dbSNP: the NCBI database of genetic variation. Nucl Acids Res 2001, 29(1):308-11.

36. Lewis $B P$, Burge $C B$, Bartel DP: Conserved seed pairing, often flanked by adenosines, indicates that thousands of human genes are microRNA targets. Cell 2005, 4(1):15-20, 120.

37. Ozsolak F, Poling LL, Wang ZX, Liu H, Liu XS, Roeder RG, Zhang XM Song SJ, Fisher DE: Chromatin structure analyses identify miRNA promoters. Genes Develop 2008, 22:3172-3183.

38. Rebecca M, Euskirchen G, Bertone P, Stephen H, Thomas ER, Luscombe NM, Rinn JL, Nelson FK, Miller P, Gerstein M, Weissman S, Snyder M: Distribution of NF-KB-binding sites across human chromosome 22. Proc Natl Acad Sci USA 2003, 100(21):12247-12252.

39. Wasserman WW, Sandelin A: Applied bioinformatics for the identification of regulatory elements. Nat Rev Genet 2004, 5(4):276-87.

doi:10.1186/1471-2164-12-72

Cite this article as: Liu et al: Analysis of nucleosome positioning determined by DNA helix curvature in the human genome. BMC Genomics 2011 12:72.

\section{Submit your next manuscript to BioMed Central} and take full advantage of:

- Convenient online submission

- Thorough peer review

- No space constraints or color figure charges

- Immediate publication on acceptance

- Inclusion in PubMed, CAS, Scopus and Google Scholar

- Research which is freely available for redistribution

Submit your manuscript at www.biomedcentral.com/submit 